\title{
Congestion Control in Manets Using Hybrid Routing Protocol
}

\author{
Gargi Parashar ${ }^{1}$, Manisha Sharma ${ }^{2}$ \\ ${ }^{\text {I}}$ (ECE Department, School of technology and Sciences/Lovely Professional University, India) \\ ${ }^{2}$ (ECE Department, School of technology and Sciences/Lovely Professional University, India)
}

\begin{abstract}
As the network size increases the probability of congestion occurrence at nodes increases. This is because of the event driven nature of ad hoc networks that leads to unpredictable network load. As a result congestion may occur at the nodes which receive more data than that can be forwarded and cause packet losses. In this paper we propose a hybrid scheme that attempts to avoid packet loss due to congestion as well as reduce end to end delay in delivering data packets by combining two protocols- Destination sequenced distance vector routing (DSDV), which is a table driven or proactive protocol and Improved Ad-hoc on demand vector routing $(I A O D V)$ which is an on-demand or reactive protocol that reduces packet loss due to congestion. The strategy adopted is use DSDV for path selection and if congestion occurs than switch over to IAODV. The routing performance of this model is then compared with IAODV and DSDV in terms of end to end delay, throughput and packet delivery fraction.
\end{abstract}

Keywords- DSDV, Hybrid protocol, AODV, IAODV, MANET

\section{Introduction}

The paradigm, where wireless nodes communicate with each other and create their own adhoc network independent of any infrastructure, is most popular these days. It is called as peer to peer communication. Routing is the most essential part of this type of communication. The IETF MANET working group is concerned with standardizing IP (layer 3) routing protocol functionality suitable for wireless ad hoc networks. There are many routing protocols described for MANETs. Some of them are reactive i.e. find route when needed and some are proactive or table-driven i.e. find routes before needed. Destination Sequence Distance Vector (DSDV) and Optimized Link State Routing (OLSR) are commonly used proactive routing algorithms while Dynamic Source Routing (DSR) and Ad hoc On-Demand Distance Vector (AODV) are the most commonly used reactive protocol. The size of ad hoc network if increases beyond a certain limited area, the use of any single routing algorithm alone may not work efficiently. Therefore the usage scenario, number of nodes in a particular network and the occupancy of buffers of a node greatly affects the choice of routing algorithm. For example the network can be as small as comprising only a few numbers of nodes in a small conference room or it can be as large as a sensor network where a great deal of nodes are needed. As the size and load of a network increases the probability of congestion and relative delay in packet delivering increases which can sometimes lead to loss of data. A hybrid routing algorithm that combines the merits of existing protocols can be used to address this issue of growth in network size and load balancing whose behavior can be modified according to the size of network.

The paper is organized as section 1 is introduction, section 2 discussion of related work, section 3 describes the proposed model, section 4 gives advantages of this scheme, section 5 presents simulation results and finally section 6 concludes the paper.

\section{RELATED WORK}

Our work involves managing the load of an adhoc network by fragmentation. The available literature has been reviewed in this context.

The Zone Routing Protocol [1] (ZRP) was the first hybrid routing protocol with both proactive and reactive routing components. ZRP defines a zone around each node consisting of its k-neighborhood. Routing within a zone is performed using a proactive routing protocol and routing in different zones is performed by an on-demand routing protocol. ZRP performs efficient route discovery through bordercasting; route requests are spread by multicasting them directly to the nodes on the border of its zone. The size of the zone is dynamically determined based on network load.

A flat mobile ad hoc network has an inherent scalability limitation in terms of achievable network capacity. It is observed that as the network size increases, per node throughput of an ad hoc network decreases. This is due to the fact that in large scale networks, flat structure of networks results in long hop paths which are prone to break because of route break or power depletion. [2] defines a hybrid protocol in which the long hop paths are avoided by using backbone power capable nodes concept working as mobile backbone network.

HCR (Hybrid Cluster Routing) is proposed in [3] where nodes are organized into a hierarchical structure of multi-hop clusters using a stable distributed clustering algorithm. Simulation results show that HCR has better 
scalability, robustness and adaptability to large scale mobile ad hoc networks compared with some well-known routing protocols, e.g. AODV, DSR, and CBRP.

AODV and OLSR are combined to form AOHR (AODV and OLSR hybrid routing) [4]. Here the characteristics of high data delivery fraction, low overheads, and short delay in AODV are combined with the characteristics of optimized routing length in OLSR, which means that AOHR is immune from topological structures.

Sharp Hybrid Adaptive Routing Protocol (SHARP), is designed to dynamically adapt to changing network characteristics and traffic behavior [5]. SHARP is driven by the fundamental trade-off between proactive dissemination and reactive discovery of routing information. It can automatically find balance point between these two strategies through an analytical model for making an informed trade-off and dynamic network measurements. It can act as a purely reactive protocol in a quiescent network, or use purely proactive routing for hosts to which routes are in wide demand. SHARP uses efficient mechanisms for dynamically manipulating the zone size and simultaneously perform fine-grained adaptation with low overhead.[6] presented another hybrid protocol scheme utilizing AODV and DSDV where each node maintains a single shared routing table for both DSDV and for AODV resulting in reduced routing load compared to DSDV and reduced packet transmission delay compared to AODV as the number of nodes grows in the network. This also significantly reduces the size of the DSDV routing table as the number of nodes grows.

Till now we have discussed various hybrid routing schemes. There are also certain enhancements in existing protocols for e.g. in [7], Improved AODV is proposed and it is compared with original AODV using IEEE 802.11 and IEEE 802.15.4 standards. This improved AODV routing protocol reset a new shortest routing path during sending packet. The simulation results showed that in case of fixed nodes both protocols give similar throughput but for moving nodes improved AODV presents good results. Also in [8], an INOVEL protocol is given which is nothing but an improved form of AODV that avoid congestion by distributing the load of the congestion affected node (CAN) to the nodes nearer to it with higher buffer occupancy. As the near sink node has a multiple routing paths towards sink, i.e. it has one primary route that connect it the sink and more than one alternate routes toward the sink from each sensor node. So INOVEL allows maximum use of alternate routes during the congestion period to avoid congestion.

The above discussion concludes that researchers have chosen two algorithms one is proactive and other reactive and combined them to produce a new model. One of the drawbacks of these protocols is that as the network size increases the performance of the network degrades. Another important thing noted is that in previous protocols no flooding control and loss of data due to congestion was considered. To overcome these drawbacks we proposed a new model combining IAODV and DSDV and its routing performance is compared with IAODV and DSDV in terms of end to end delay, throughput and packet delivery fraction.

\section{Proposed Hybrid Protocol Model}

In this proposed model packet loss due to congestion and excessive load at intermediate nodes is tried to be reduced. Balancing load to avoid congestion inside novel scheme of flow control is actually performed by creating a cycle on a node where the congestion probability is high i.e. at near sink node to find all those nearer nodes where buffer occupancy is high. Near sink node and nodes nearer to it contains the routing table including information about its own I.P. address, I.P. address of nearer neighbor nodes, distance between the nodes, \& queue length of each node as shown in Fig.1

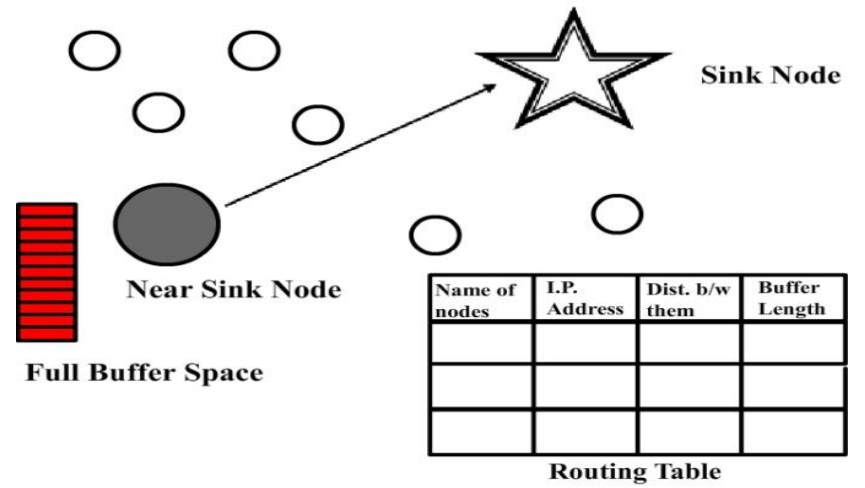

Fig.1 Congestion Affected Node with its Routing table

The dynamic nature of wireless sensor network cause the topology to automatically change due to change in topology each node automatically updates its information in its own routing table \& the routing table of the nearer node regarding its buffer length, its distance from other nodes, its I.P. address There are following steps to be followed during this process: 
1. Firstly a proactive/table driven algorithm is applied to find the path from source node to destination node. If path is congestion free than data packets are delivered as such following the defined route. If congestion occurs than following technique is followed.

2. A Hop-by-Hop algorithm is implemented on congestion affected node. This algorithm check's the routing table of congestion affected node to find all the nodes nearer to it with minimum response length time i.e. it must have maximum buffer occupancy to accommodate the load

3. A Hop-by-Hop algorithm is implemented on congestion affected node. This algorithm check's the routing table of congestion affected node to find all the nodes nearer to it with minimum response length time i.e. it must have maximum buffer occupancy to accommodate the load of congestion affected node.

4. After finding the node with free buffer space Hop-by-Hop algorithm make that node as the child node of the congestion affected node and the alternate routes from the congestion affected node to the nearer node will become active to transmit data so as to utilize its buffer space.

5. As soon as the time period during which child node receives the packets in its buffer space from the congestion affected parent node it will store them in its buffer for a short time interval.

6. On receiving the packets from congestion affected node by the nearer node at the same time this node will immediately implement the Hop-to-Destination algorithm to forward the packets to the destination i.e. sink node base station within the finite amount of time. The scenario for this whole process is shown in Fig.2.

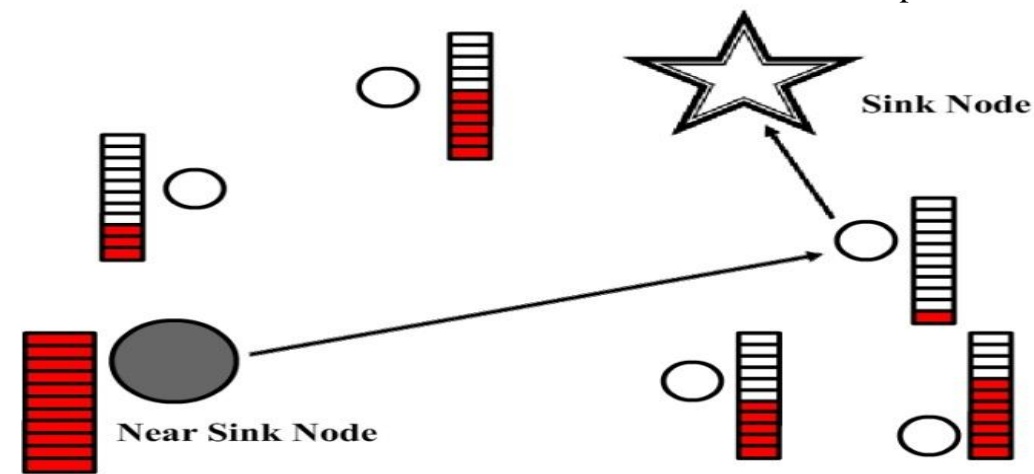

Full Buffer Space

Fig. 2 Congestion Affected Node with its Routing table

The proposed model combines the working of both IAODV and DSDV. When a source node in an ad hoc network attempts to send data to some destination node, the route finding is done using DSDV routing protocol which allows each node to maintain a routing table in advance. Whenever a node initiates communication, it can refer to its routing table for the route. This process is used until and unless there is no congestion in the path. Whenever some congestion arrives the proposed scheme inclined towards using IAODV to find path which allows safe delivery of data packets without any packet dropping. This strategy can be more clearly explained with the help of a flowchart as given in Fig.3.

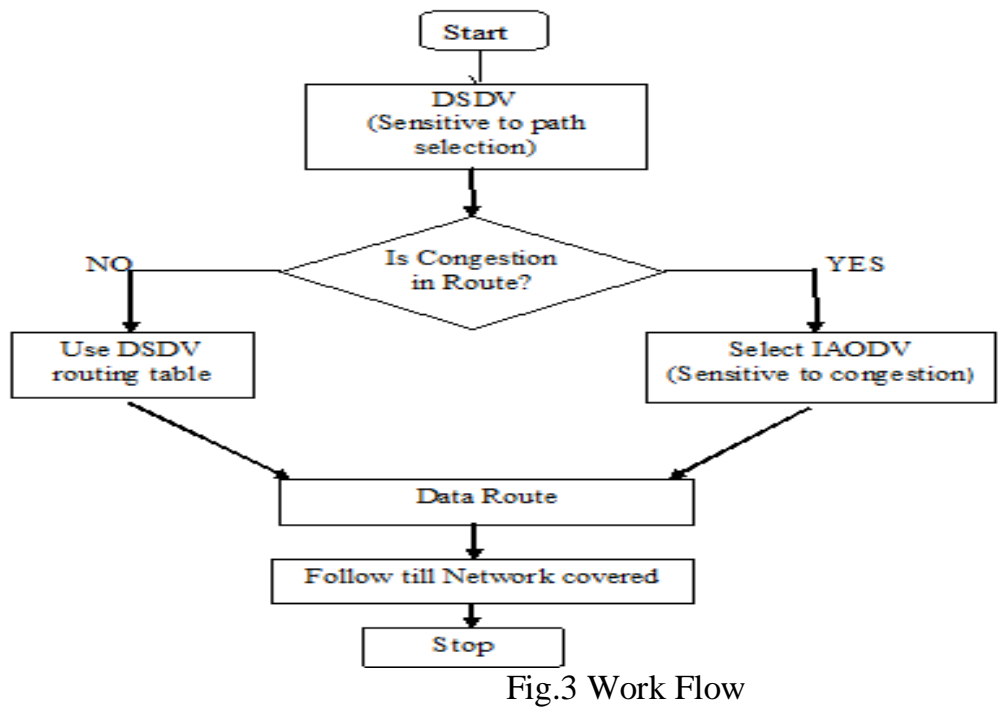




\section{Advantages Of Proposed Model}

1. DSDV is best for small to medium size networks. As the network size increases the performance of DSDV routing algorithm decreases. To overcome this problem in our approach nodes are made to switch to follow IAODV as network grows and probability of congestion increases. Thus performance can be improved even with large networks.

2. One distinct advantage of our model is that when a node on the route moves out or fails, instead of discarding the whole original route and discovering a new route from source to destination, only the congestion affected node has to find new path.

3. Another distinct advantage of our model as compared to DSDV is that packet dropping rate is reduced. Thus maximum packet delivery fraction can be achieved.

4. This proposed model attempts to maximize the utilization of free nodes and there buffer space. Thus optimization of network resources can be achieved.

5. Another advantage of this model is reduction of end to end delay

\section{Simulation And Performance Analysis}

The simulation is conducted using Network Simulator tool NS-2 version 2.34. The simulation environment has been conducted with the LINUX operating system, because NS-2 works with Linux platform only. NS-2 accepts as input a scenario file that describes the exact motion of each node and the exact packets originated by each node, together with the exact time at which each change in motion or packet origination is to occur. The detailed trace file created by each run is stored to disk, and analyzed using a variety of scripts, particularly one called file *.tr that counts the number of packets successfully delivered and the length of the paths taken by the packets, as well as additional information about the internal functioning of each scripts executed. This data is further analyzed with AWK file to produce the graphs. The list of various parameters taken are given in Table1 below

\begin{tabular}{|l|l|}
\hline Parameter & Value \\
\hline Number of Nodes & 50 \\
\hline Simulation Time & $50 \mathrm{sec}$ \\
\hline Pause Time & $10 \mathrm{sec}$ \\
\hline Packet size & 512 bytes \\
\hline Channel Type & Wireless \\
\hline Queue length & 50 \\
\hline Bandwidth & $40 \mathrm{MHz}$ \\
\hline Area of the Network & $1000 \mathrm{~m}$ X 1000m \\
\hline Traffic types & CBR/UDP, FTP/ \\
\hline Simulator & Ns-2.34 \\
\hline Mobility model & Random Waypoint \\
\hline
\end{tabular}

Table 1 List of general parameters used in all simulations

While comparing IAODV and DSDV with the proposed hybrid protocol, we focused on three performance measurements- average end to end delay, throughput and packet delivery fraction.

(i) Average End to end delay of data packets: The average time from the beginning of a packet transmission at a source node until packet delivery to a destination. Calculate the send(S) time (t) and receive (R) time (T) and average it. Fig.4 shows the xgraph between IAODV,DSDV and hybrid protocol at a pause time set to10sec.

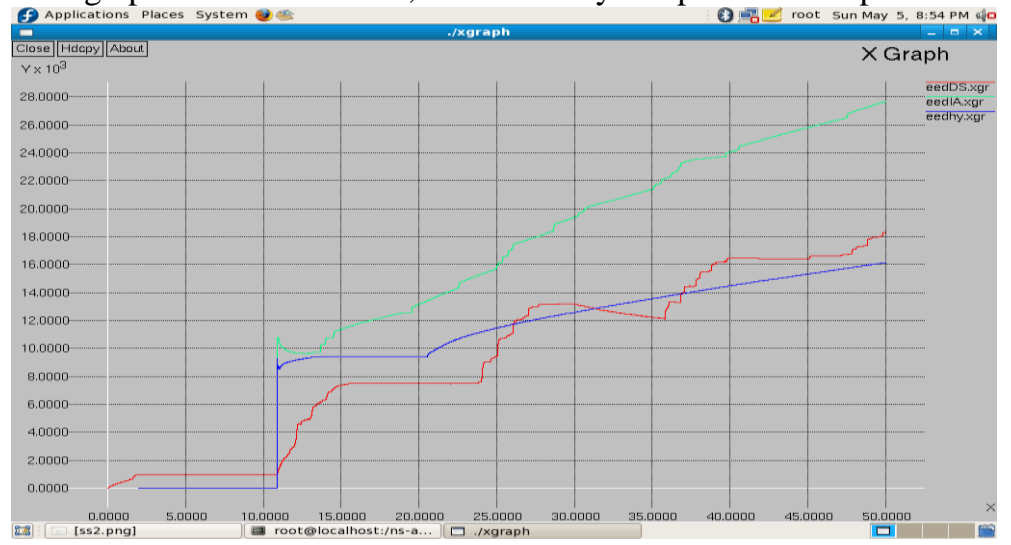

Fig.4 A Screen shot of graph showing comparison of end to end delay between DSDV, IAODV and Hybrid Protocol 
ii) Packet Delivery fraction- The ratio of the number of data packets successfully delivered to the destinations to those generated by source. Packet delivery fraction $=$ (Received packets/Sent packets)*100. Fig.5 shows a comparison between both the routing protocols on the basis of packet delivery fraction as a function of pause time and using different types of traffic sources.

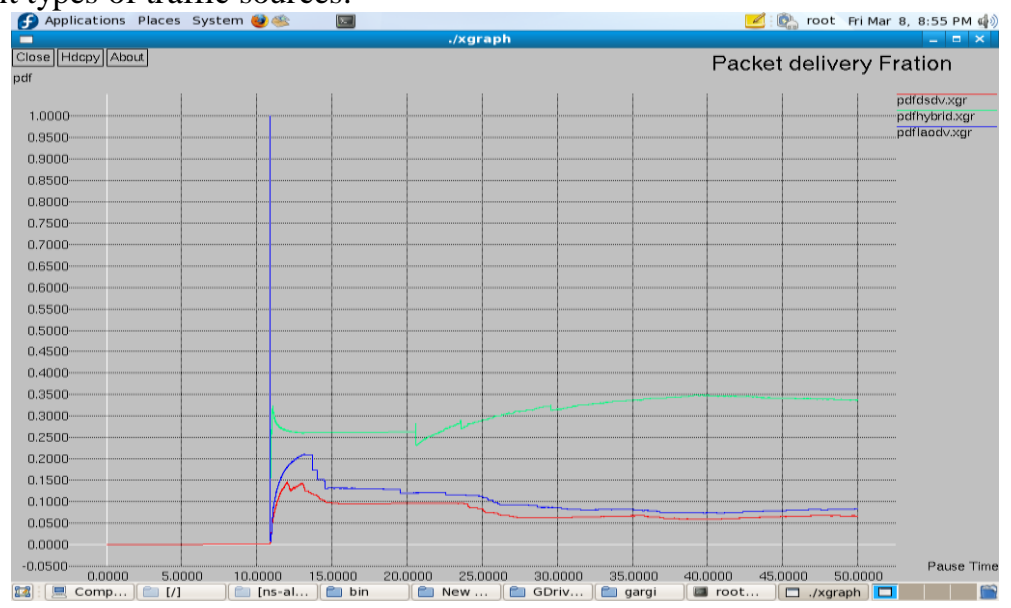

Fig.5 A Screen shot of graph showing comparison of Packet Delivery Fraction between DSDV, IAODV and Hybrid Protocol

iii) Throughput- The ratio of the total amount of data that reaches a receiver from a sender to the time it takes for the receiver to get the last packet is referred to as throughput. It is expressed in bits per second or packets per second. Fig.6 shows throughput of hybrid protocol, IAODV and DSDV

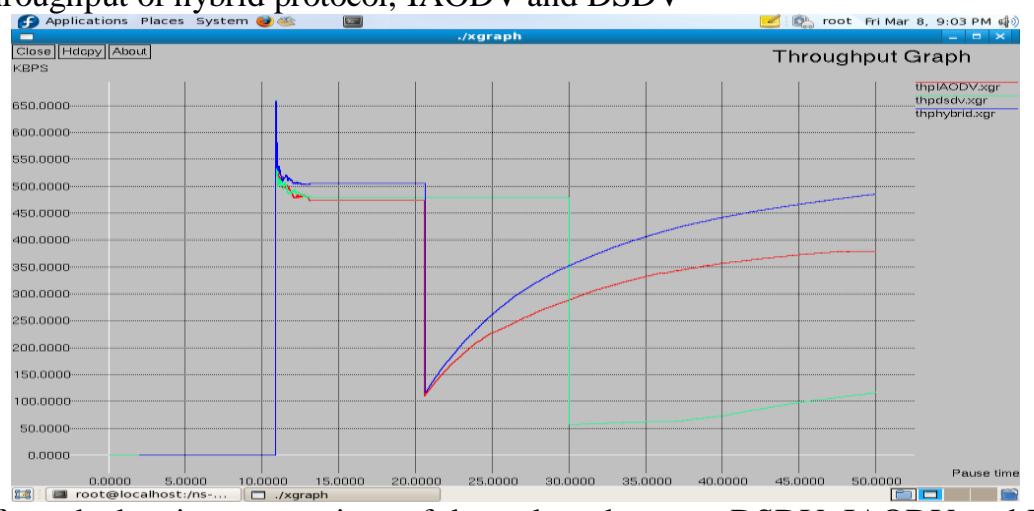

Fig.6 A Screen shot of graph showing comparison of throughput between DSDV, IAODV and Hybrid Protocol Screenshot

To analyze these graphs in more general way average results are taken. Average results are the obtained by taking the average of performance matrix obtained over whole simulation environment. In NS- 2 the command "awk $-\mathrm{f}$ " is used to find out the average results. The average result of above three performance matrices in terms of the described three protocols as per the configuration taken in this thesis is given below:-

\begin{tabular}{|l|l|l|l|}
\hline PROTOCOL & $\begin{array}{l}\text { End to end } \\
\text { delay(in } \\
\text { ms) }\end{array}$ & PDF & $\begin{array}{l}\text { Throughput } \\
\text { (in Kbps) }\end{array}$ \\
\hline IAODV & 27.676584 & 0.08 & 211.26 \\
\hline DSDV & 18.300936 & 0.07 & 116.62 \\
\hline Hybrid & 16.165742 & 0.34 & 485.35 \\
\hline
\end{tabular}

Table 2 Average results of end to end delay, pdf and throughput

These results given above show that end to end delay of DSDV is lesser than IAODV. This is due to the proactive or table driven nature of DSDV which avoid the time consumed to find out the path from source to destination as it can derive from the routing table of DSDV. However, in case of proposed hybrid scheme, it proves to be better than DSDV as it further reduces the delay by rather finding new routes from source to destination in case of congestion, calculates it only from congestion affected node.

Packet Delivery fraction of IAODV is obtained higher than DSDV. This is in line with the theory presented in previous chapters. But there is only marginal improvement of 0.01. In case of proposed Hybrid scheme there is 
tremendous improvement in pdf as this protocol ensures better delivery of data packets even in case of congestion.

Throughput of proposed hybrid scheme is also relatively much better than DSDV and IAODV. While when DSDV and IAODV are compared throughput of IAODV is obtained higher than DSDV.

The above results show that the performance of proposed hybrid scheme is far better than IAODV and DSDV.

\section{CONCLUSION AND FUTURE WORK}

The proposed model in our work is an innovative way to deal with congestion along with reduction in time taken for transmission. This proposed model is efficient for using the network resources in an optimized manner by distributing load among buffers of neighboring nodes of congestion affected node while simultaneously decreasing end to end delay. Simulation results show improvement in performance in terms of high packet delivery fraction and throughput.

Currently, the work is done on unicast protocols only. The future work involves implementing this scheme for multicasting also and then analyzing its performance. Also reduction in power consumption and security threats this scheme can create over network will be investigated.

\section{REFERENCES}

[1] E. Topalis S. Giannoulis, C. Antonopoulos and S. Koubias. Zrp versus DSR and TORA: A Compressive Survey on ZRP Performance. 10th IEEE Conference, ETFA 2005, Sept 2005.

[2] Kush, A., Gupta, P.; Hwang, C.J. A hybrid stable routing protocol for mobile ad hoc networks. Global Mobile Congress, 12-14 Oct. 2009

[3 ]Xiaoguang Niu Zhihua Tao, Gongyi Wu, Changcheng Huang,Li Cui ,Hybrid Cluster Routing: An Efficient Routing Protocol for Mobile Ad Hoc Networks. IEEE International Conference on Communications, 2006. ICC'06, Vol. 8, 3554-3559

[4] Wu Shaochuan Tan Xuezhi; Jia Shilou. AOHR: AODV and OLSR Hybrid Routing Protocol for Mobile Ad Hoc Networks, International Conference on Communications, Circuits and Systems Proceedings, June 2006 Vol. 3,1487-1491

[5] Venugopalan Ramasubramanian Zygmunt J. Haas Emin Gun Sirer SHARP: A Hybrid Adaptive Routing Protocol for Mobile Ad Hoc Networks.

[6] Minseok Oh, A Hybrid Routing Protocol and Performance Evaluation in a Wireless Mesh Network, Journal of Advanced Information technology and convergence, vol.1, No.1, July 2011

[7] Yu-Doo Kim, Il-Young Moon, Sung-Joo Cho, A comparison of improved AODV routing protocol based on IEEE 802.11 and IEEE 802.15.4, Journal of Engineering Science and Technology Vol.4,No.2,2009.

[8] Harwinder Singh Sohal, Rajbir Singh Cheema, Ankit Arora, Improved NOVEL Routing Protocol for Load Balancing in Wireless Sensor Network, IJCST Vol. 2, Issue 3, September 2011. 